\title{
Reliable Communication using Erasure Codes for Monitoring Bats in the Wild
}

\author{
Margit Mutschlechner, Florian Klingler, Felix Erlacher, Florian Hagenauer, Marcel Kiessling and Falko Dressler \\ Computer and Communication Systems, Institute of Computer Science, University of Innsbruck, Austria \\ \{mutschlechner, klingler, erlacher, hagenauer, kiessling, dressler\}@ccs-labs.org
}

\begin{abstract}
We show the feasibility of using Erasure Codes (ECs) for reliable communication in strongly energy-constrained sensor network applications. In particular, we aim at observing group dynamics of bats in their natural habitat. Bats are to be equipped with ultra-light sensor nodes to continuously monitor contacts between each other and to track their flights. This data needs to be uploaded to a stationary ground network whenever a bat comes into communication range. As the quality of this communication link is fluctuating, reliability improvement techniques need to be implemented. ECs allow to enhance the reliability of data transmissions with only a marginal overhead. After showing the improvements using ECs in simulation, we now implemented the full system using a prototype platform to study the general feasibility in a more realistic environment.
\end{abstract}

\section{INTRODUCTION}

The use of sensor networking technology for wildlife monitoring already has quite some tradition, especially for non-intrusive long-term observations. The first projects relied on comparably large prototype sensor platforms. Examples include the Great Duck Island [1] or ZebraNet [2]. In more recent activities, heterogeneous sensor nodes have been used for tracking generic animals and endangered species such as Iberian lynx in the surrounding area of wildlife passages, which was built to establish safe ways for animals to cross transportation infrastructures [3]. This system allows target identification through the use of video sensors connected to strategically deployed nodes. From these successful approaches to wildlife monitoring using sensor networks, the community learned about hardware design issues, network management, and data collection techniques.

In the new BATS project ${ }^{1}$ on monitoring the group dynamics of bats in their natural habitat, we go one step further and investigate potentials of ultra-low power sensor systems carried by the bats to monitor contacts between individuals and to track their routes. The aim of the project is to support biologists with their study on bats, one of the most protected species in European Union, to track their living habitats and social behaviors. Mouse-eared bats (Myotis myotis) are the main target [4]. The key challenge is that the animals weighting about $20 \mathrm{~g}$ can carry sensors of at most $2 \mathrm{~g}$, which strongly limits the available energy budget.

The scenario employs mobile sensor nodes which are situated on bats and base nodes forming a stationary ground network as

\footnotetext{
${ }^{1}$ Dynamically adaptive applications for bat localization using embedded communicating sensor systems, http://www.for-bats.org/; This work has been supported by the German Research Foundation (DFG), grant no. FOR 1508.
}

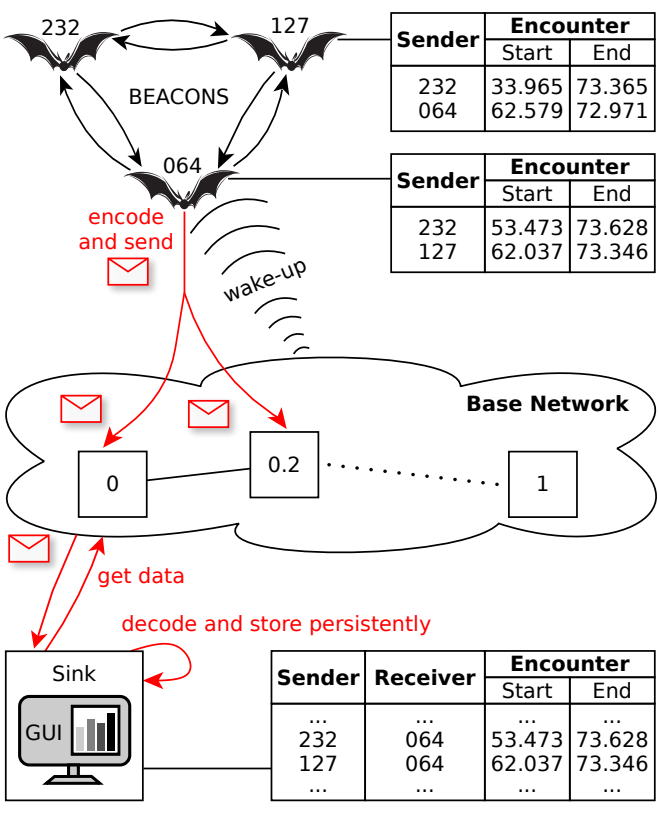

Figure 1: The BATS communication scenario.

shown in Figure 1. Mobile nodes continuously exchange contact information but only appear in the communication range of a base station on an irregular basis. If in communication range, they are supposed to upload all stored contact information. Once the data has been received by a base node it has to be stored temporarily inside the base network up until being requested by any of the nodes for further processing.

The most critical challenge is the channel quality, which may vary quickly due to the continuous movements of bats and the heterogeneous environment. Thus, the communication is in general assumed to be highly unreliable. Conventional reliability improving approaches such as full data replication or on-demand retransmission are too expensive or even not possible due to very strict network lifetime constraints [5].

We propose the use of Erasure Codes (ECs) to improve the communication reliability between the mobile nodes and the base network [6]. ECs are Forward Error Corrections (FECs) for the erasure channel that enhances data transmission reliability by introducing redundancy, however, without the overhead of strict replication. The encoding process at the sender extends a group of $k$ original packets to $n$ packets by generating $m=$ $n-k$ linearly independent redundant packets. The decoding process at the receiver reverses this process if any $k^{\prime} \geq k$ out 


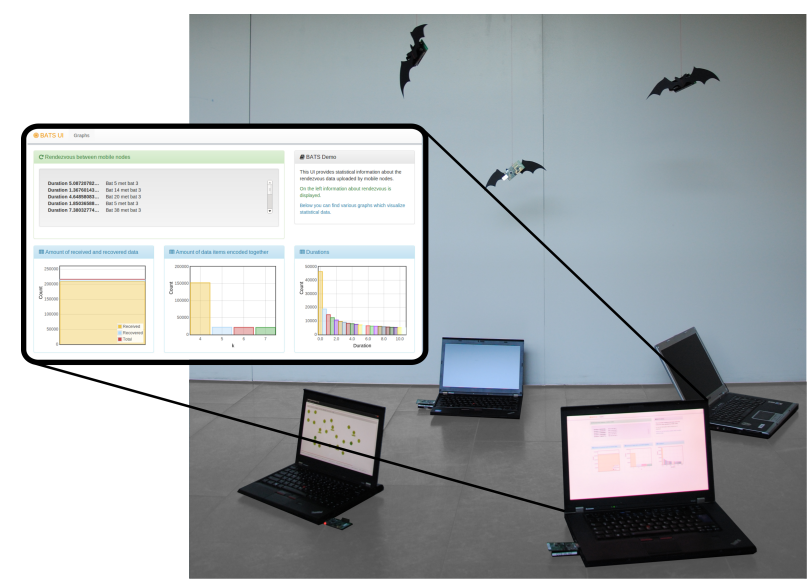

Figure 2: Demo setup showing mobile sensors collecting contact information and ground nodes serving as data sinks.

of $n$ packets were successfully transmitted and is, therefore, able to recover the $k$ original packets. As we have shown in previous work [7], ECs offer a better performance with reduced costs compared to the simplistic approach of sending replica together with the original data.

In this demo, we present now a prototype implementation of the presented system based on off-the-shelf sensor nodes and standard laptop computers, since the target hardware is still under development at this moment. We improve the data transmission reliability with a Reed-Solomon code [8], an optimal EC with $k^{\prime}=k$ showing the best performance according to our previous work [7].

\section{EXPERIMENT SETUP}

The experiment setup is depicted in Figure 2. The system consists of three types of nodes. Mobile nodes, envisioned to be situated on bats, are realized with battery-powered TelosB sensor nodes. The base nodes, envisioned to be laid out in the habitat on the ground to form a stationary backbone network, are replaced by laptop computers for this demo. For demonstration purpose, one dedicated laptop acts as sink periodically pulling information from the base network and displaying collected information and statistical data.

Mobile nodes periodically broadcast beacons of one byte containing their unique identifiers to inform nearby mobile nodes about their existence. The interval between two beacons was set to be $1 \mathrm{~s}$. Upon reception of a beacon, the receiving mobile node stores this information to build up a rendezvous table. Two beacons are considered to be received continuously if the difference of their arrival time is below a pre-defined threshold, which is set to twice the beacon sending interval.

With the envisioned use of multi-stage wake-up transceivers a mobile node powers-up the radio and initializes the data transmission when it is located within the transmission range of at least one base node. Since such a hardware platform is not available at this moment, we imitate the wake-up signal by a button push. We use the aforementioned Reed-Solomon code for the reliable transmission of this data, as the loss of complete contact information would be critical to the overall system's lifetime (data would have to be retransmitted accordingly). Since each row of the rendezvous table is sent in an individual packet, the number of table entries has to reach the predefined threshold $k$ for encoding. A data packet has a size of $17 \mathrm{~B}$, where $9 \mathrm{~B}$ are reserved for the payload containing either one table entry or redundant data produced by the encoding algorithm. The remaining overhead is formed by the mobile node's identifier of $1 \mathrm{~B}$ and the needed metadata of the EC.

Since all packets might be transmitted to different ground nodes, there is the need for an efficient and decentralized distributed data storage and lookup. A promising concept are Distributed Hash Tables (DHTs) that are integrated with the routing protocol [9], [10]. There exist various protocols for sensor networks providing standard DHT functionalities, however, most of them rely on globally valid topology information, need geographic location information, or do not take into consideration the physical position of nodes, which leads to increased routing paths. We selected the Virtual Cord Protocol (VCP) [10], which overcomes these shortcomings. The nodes form a cord based on neighborhood information, where each node is assigned a unique value in the range $[0,1]$. Each node is then responsible for data with a hash value that matches its virtual node identifier.

Finally, for demonstration purposed, we configured one laptop to act as sink to periodically pull temporarily stored data from the base network. After decoding missing data packets it persistently stores the information and visualizes the data together with statistics on a web interface.

\section{REFERENCES}

[1] A. Mainwaring, J. Polastre, R. Szewczyk, D. Culler, and J. Anderson, "Wireless Sensor Networks for Habitat Monitoring," in ACM Workshop on Wireless Sensor Networks and Applications, Atlanta, GA, September 2002.

[2] P. Juang, H. Oki, Y. Wang, M. Martonosi, L.-S. Peh, and D. Rubenstein, "Energy-Efficient Computing for Wildlife Tracking: Design Tradeoffs and Early Experiences with ZebraNet," ACM SIGOPS Operating Systems Review, vol. 36, no. 5, pp. 96-107, December 2002.

[3] A.-J. Garcia-Sanchez, F. Garcia-Sanchez, F. Losilla, P. Kulakowski, J. Garcia-Haro, A. Rodr'iguez, J.-V. L'opez-Bao, and F. Palomares, "Wireless Sensor Network Deployment for Monitoring Wildlife Passages," Sensors, vol. 10, no. 8, pp. 7236-7262, 2010.

[4] B.-U. Rudolph, A. Liegl, and O. Von Helversen, "Habitat Selection and Activity Patterns in the Greater Mouse-Eared Bat Myotis myotis," Acta Chiropterologica, vol. 11, no. 2, pp. 351-361, 2009.

[5] I. Dietrich and F. Dressler, "On the Lifetime of Wireless Sensor Networks,' ACM Transactions on Sensor Networks, vol. 5, no. 1, pp. 1-39, February 2009.

[6] M. G. Luby, M. Mitzenmacher, M. A. Shokrollahi, and D. A. Spielman, "Efficient Erasure Correcting Codes," IEEE Transactions on Information Theory, vol. 47, no. 2, pp. 569-584, 2001.

[7] M. Mutschlechner, B. Li, R. Kapitza, and F. Dressler, "Using Erasure Codes to Overcome Reliability Issues in Energy-Constrained Sensor Networks," in IEEE/IFIP WONS 2014. Obergurgl, Austria: IEEE, April 2014, to appear.

[8] L. Rizzo, "Effective Erasure Codes for Reliable Computer Communication Protocols," ACM SIGCOMM Computer Communication Review, vol. 27, no. 2, pp. 24-36, April 1997.

[9] M. Caesar, M. Castro, E. B. Nightingale, G. O'Shea, and A. Rowstron, "Virtual Ring Routing: Network routing inspired by DHTs," in $A C M$ SIGCOMM 2006. Pisa, Italy: ACM, September 2006.

[10] A. Awad, R. German, and F. Dressler, "Exploiting Virtual Coordinates for Improved Routing Performance in Sensor Networks," IEEE Transactions on Mobile Computing, vol. 10, no. 9, pp. 1214-1226, September 2011. 